\title{
On the connectivity of graphs embedded in surfaces II
}

\author{
Michael D. Plummer \\ Department of Mathematics \\ Vanderbilt University \\ Nashville, TN 37240, USA \\ michael.d.plummer@vanderbilt.edu \\ Xiaoya Zha* \\ Department of Mathematical Sciences \\ Middle Tennessee State University \\ Murfreesboro, TN 37132, USA \\ xzha@mtsu.edu
}

Submitted: February 6, 2002; Accepted: September 12, 2002

MR Subject Classification: 05C10, 05C40

\begin{abstract}
Let $\kappa_{\max }(\Sigma)$ denote the maximum value for the connectivity of any graph which embeds in the topological surface $\Sigma$. The connectivity interval for $\Sigma$ is the set of integers in the interval $\left[1, \kappa_{\max }(\Sigma)\right]$. Given an integer $i$ in $\left[1, \kappa_{\max }(\Sigma)\right]$ it is a trivial problem to demonstrate that there is a graph $G_{i}$ with connectivity $i$ which also embeds in $\Sigma$. We will say that one can saturate the connectivity interval in this case.

Note that no restrictions have been placed on the embeddings in the above problem, however. What if we demand that the embeddings in question be 2-cell or even that they be genus embeddings?

The problem of saturating the connectivity interval for 2-cell embeddings will be solved completely in the present work. In connection with the apparently much harder saturation question for genus embeddings, it will be shown that one can always saturate the subinterval $\left[1,\left\lfloor 0.7 \kappa_{\max }(\Sigma)\right\rfloor\right]$.
\end{abstract}

\footnotetext{
* work supported by NSF Grant DMS-9622780 and Middle Tennessee State University Faculty Research Grant 1999
} 


\section{Introduction}

In his 1973 paper, Cook [C1] studied the relation between the connectivity of a graph and the surfaces into which it can be embedded. He proved that the following result holds for all surfaces except the plane:

$$
\kappa(G) \leq\left\lfloor\frac{5+\sqrt{49-24 \chi}}{2}\right\rfloor,
$$

where $\kappa(G)$ denotes the vertex connectivity of graph $G$ and $\chi$ is the Euler characteristic of any surface in which $G$ embeds. Moreover, Cook showed that these bounds are attained by complete graphs in all cases except the Klein bottle.

In [PZ1] we explored in more detail relations between the connectivity of embedded graphs and the surfaces in which they are embedded. Let $\kappa_{\max }(\Sigma)$ denote the maximum connectivity among all graphs which embed in $\Sigma$ and let $\kappa_{g e n}(\Sigma)$ be the maximum connectivity among all graphs which genus embed in $\Sigma$. It was shown that, somewhat surprisingly, $\kappa_{g e n}$ is not a monotone non-decreasing function of the genus. Also for some surfaces, $\kappa_{g e n}$ is strictly less than $\kappa_{\max }$. In fact it was proved that such so-called Class $B$ surfaces are not only infinite in number, but that they must occur infinitely often periodically as the genus parameter increases to infinity. It was also shown that in the case when the complete graph which attains Cook's maximum connectivity bound actually genus embeds in the surface, that with a finite number of exceptions, it is the unique graph attaining this bound.

Let us begin the present work by considering a very easy problem. If $G_{\max }$ is a graph which embeds in a surface $\Sigma$ and $\kappa\left(G_{\max }\right)=\kappa_{\max }$ for that surface, then by removing edges all incident with a common vertex one by one, it is trivial to construct a sequence of graphs $G_{r}=G_{\max }, G_{r-1}, \ldots, G_{2}, G_{1}$ such that $\kappa\left(G_{i}\right)=i$ and each $G_{i}$ embeds in the surface. In this case, we say that we can saturate the connectivity interval $\left[1, \kappa_{\max }\right]$.

But note that nothing was said about the nature of the embeddings along the way. In particular, it was not demanded that they be 2-cell or that they be genus embeddings. In the present paper, the focus will be on two questions:

Question 1: Given a surface $\Sigma$ and an integer $i$, does there exist a graph $G$ with $\kappa(G)=i$ such that $G$ 2-cell embeds in $\Sigma$ (i.e., each face is homeomorphic to an open disk)?

Question 2: Let $\Sigma$ and $i$ be as in Question 1. Does there exist a graph $G$ with $\kappa(G)=i$ such that $G$ genus embeds in $\Sigma$ ?

With the aid of theorems by Duke [D1] and Stahl [S1], we will answer Question 1 completely. The solution is not difficult. Regarding the second question, it will be shown that for $i \in\left[1,\left\lfloor 0.7 \kappa_{\max }\right\rfloor\right]$, Question 2 has a positive answer.

In fact the second question appears to be quite difficult. For one thing, all embeddings sought must be genus embeddings and the genus problem for graphs is known to be NP-complete. (See [T1].) Thus one needs to construct special genus embeddings with the required connectivity the genera of which are easy to determine. Also for 
certain values of $i$ in Question 2, an affirmative answer to the question turns out to imply an answer to an unsolved problem of long standing. Let $O(m)$ denote the generalized octahedron graph which is obtained from the complete graph $K_{m}$ by deleting a maximum matching. When $m$ is even and $m / 2 \equiv 0,1(\bmod 3)$, the orientable genus of $O(m)$ is known. (See [AG1] and [JR1].) Determination of the orientable genus of $(O(m))$ remains open for the remaining values of $m$.

Let $i$ in Question 2 be the connectivity of $O(m)(=m-2)$. Then by Theorems 5.1 and 5.2 of [PZ1], this special case of Question 2 actually implies the answer to the problem of determining the orientable genus of $O(m)$ for certain of the remaining values of $m$. This will be explained in more detail in the final section of the present paper.

Most of the proofs to follow involve constructions of graphs and embeddings such that (1) the embeddings are genus embeddings and (2) the graphs have the correct connectivity.

\section{Saturation of the Connectivity Interval for 2-cell Embeddings}

In this section, we proceed to answer Question 1 of the Introduction. As usual, $\gamma(G)$ (respectively, $\bar{\gamma}(G)$ ) will denote the orientable (respectively, non-orientable) genus of graph $G$. An embedding of a graph $G$ in a surface $S$ is said to be a 2-cell embedding if every face is homeomorphic to an open disk. The maximum orientable (respectively maximum non-orientable) genus $\gamma_{M}(G)$ (respectively $\bar{\gamma}_{M}(G)$ ) of graph $G$ is the largest genus of any orientable surface $S$ (respectively, non-orientable surface $N$ ) in which $G$ has a 2-cell embedding.

The following results due to Duke [D1] in the orientable case and to Stahl [S1] in the non-orientable case guarantee that there is a 2-cell embedding of $G$ in all surfaces with genera values between and including those of the minimum and maximum surfaces.

Theorem 2.1. If $\gamma(G) \leq \gamma \leq \gamma_{M}(G)$ ( or if $\bar{\gamma}(G) \leq \bar{\gamma} \leq \bar{\gamma}_{M}(G)$ ), then there is a 2-cell embedding of $G$ in the orientable surface of genus $\gamma$ (respectively, in the non-orientable surface of genus $\bar{\gamma}$ ).

Let $G$ be a connected graph and let $T$ be an arbitrary spanning tree of $G$. The deficiency $\xi(G, T)$ of spanning tree $T$ is the number of components of $G-E(T)$ which have an odd number of edges. The deficiency $\xi(G)$ of graph $G$ is the minimum of $\xi(G, T)$ over all spanning trees $T$. Finally, let $\beta(G)$, called the Betti number or cyclomatic number of a connected graph $G$ be defined by $\beta(G)=|E(G)|-|V(G)|+1$.

Xuong [X1] and Edmonds [E2] have characterized the maximum orientable and non-orientable genus of a graph $G$ in terms of its Betti number and its deficiency.

Theorem 2.2. If $\gamma_{M}(G)\left(\bar{\gamma}_{M}(G)\right), \xi(G)$ and $\beta(G)$ represent the maximum orientable (non-orientable) genus of a connected graph $G$, the deficiency of $G$ and the Betti number of $G$ respectively, then $\gamma_{M}(G)=(1 / 2)(\beta(G)-\xi(G))$ and $\bar{\gamma}_{M}(G)=\beta(G)$. 
We make use of Theorem 2.2 in the next result which states that one can saturate the interval of connectivity $\left[1, \kappa_{\max }\right]$ with 2-cell embeddings for any surface orientable or non-orientable. Thus Question 1 in Section 1 has a complete affirmative answer.

Theorem 2.3. If $\Sigma$ is any surface, orientable or non-orientable, then for any integer $i$ in the interval $\left[1, \kappa_{\max }(\Sigma)\right]$, there is a graph $G$ with connectivity $i$ which 2-cell embeds in $\Sigma$.

Proof: First consider the orientable case. Let $S_{g}$ be any orientable surface, let $i$ be any integer in the interval $\left[1, \kappa_{\max }\left(S_{g}\right)\right]$, and let $K_{m}$ be the largest complete graph which embeds in $S_{g}$. Note that we do not assume that this embedding is necessarily 2-cell. If $g=0$ then the surface is the sphere, $\kappa_{\max }$ (sphere) $=5$, and the theorem is trivial. If $g=1$ then the surface is the torus, and $\kappa_{\max }($ torus $)=6$. Negami [N1] has shown that there are 6-connected triangulations of the torus (which therefore are 2-cell embeddings). We shall refer to these graphs as "Negami graphs". It is an easy matter to modify one of his graphs to obtain graphs with $\kappa=1, \ldots, 5$ which also 2 -cell embed on the torus.

So henceforth we will assume that $g \geq 2$ and $m \geq 8$. Suppose $i$ is an integer in the connectivity subinterval $[1, m-1]$. Let $G_{i}$ be a graph obtained from $K_{m}$ by deleting $m-i-1$ edges all incident with a common vertex $v$. Then $\kappa\left(G_{i}\right)=m-(m-i-1)-1=i$.

Clearly $\gamma\left(G_{i}\right) \leq \gamma\left(K_{m}\right) \leq g<\gamma\left(K_{m+1}\right)$. We now show that $\gamma_{M}\left(G_{i}\right) \geq \gamma\left(K_{m+1}\right)$. By Theorem 2.2, $\gamma_{M}\left(G_{i}\right)=(1 / 2)\left(\beta\left(G_{i}\right)-\xi\left(G_{i}\right)\right)$. Moreover, $\beta\left(G_{i}\right)=1-m+[m(m-$ 1) $/ 2-(m-i-1)]=m(m-1) / 2-2 m+i+2$.

It is easy to construct a spanning tree $T$ of $G_{i}$ such that $G_{i}-E(T)$ consists of only one component. (Such a tree is called a splitting tree; see White [W1].) Therefore $\xi\left(G_{i}\right)=0$ if $G-E(T)$ has an even number of edges and $\xi\left(G_{i}\right)=1$ if $G-E(T)$ has an odd number of edges. Thus $\gamma_{M}\left(G_{i}\right) \geq(1 / 2)\left(\beta\left(G_{i}\right)-1\right)=(1 / 2)(m(m-1) / 2-2 m+i+2)=$ $m(m-1) / 4-m+(1 / 2) i+1>m(m-1) / 4-m+1$. So

$$
\begin{gathered}
\gamma_{M}\left(G_{i}\right)-\gamma\left(K_{m+1}\right)>\frac{m(m-1)}{4}-m+1-\left\lceil\frac{(m-2)(m-3)}{12}\right\rceil \\
\geq \frac{m(m-1)}{4}-m+1-\frac{(m-2)(m-3)}{12}-1 \\
=\frac{m^{2}-5 m-3}{6}>\frac{(m-6)(m+1)}{6}>0,
\end{gathered}
$$

since $m \geq 8$.

Thus $\gamma_{M}\left(G_{i}\right) \geq \gamma\left(K_{m+1}\right) \geq g$ and hence $\gamma\left(G_{i}\right) \leq \gamma\left(K_{m}\right)<\gamma\left(K_{m+1}\right) \leq \gamma_{M}\left(G_{i}\right)$. Thus by Theorem 2.1, $G_{i}$ has a 2-cell embedding for every genus in the interval $\left[\gamma\left(G_{i}\right), \gamma_{M}\left(G_{i}\right)\right]$ which includes the interval $\left[\gamma\left(K_{m}\right), \gamma\left(K_{m+1}\right)\right]$ which in turn includes the genus $g$. This completes the proof in the orientable case.

The proof for the non-orientable case parallels that for the orientable case. 


\section{Constructions $I A, I B$ and $I C$ - the Orientable Case}

Starting in this Section, we will address the more difficult Question 2. We will construct some simple graphs together with genus embeddings thereof and for each of these constructions, we will derive the genus and the connectivity. Let $\gamma(s, k)$ denote the orientable genus of $K_{m}$ where $m=12 s+k, s \geq 1$ and $0 \leq k \leq 11$. For each value of $k=$ $0, \ldots, 11$ and for each $s \geq 1$ we define the function $l_{k}(s)$ by $l_{k}(s)=2[\gamma(s, k+1)-\gamma(s, k)]$ for $k \neq 2,5$, and $l_{k}(s)=2[\gamma(s, k+1)-\gamma(s, k)]+2$ for $k=2,5$, Then by the RingelYoungs Theorem [R1], $\gamma(s, k)=12 s^{2}+2 s k-7 s+\left\lceil\left(k^{2}-7 k+12\right) / 12\right\rceil$ and it follows that

$$
l_{k}(s)= \begin{cases}4 s, & \text { if } k=0,1,2,3,6, \\ 4 s+2, & \text { if } k=4,5,7,8,9,10,11\end{cases}
$$

It is known [R1] that when $k=2,5$, the graph $K_{m}-K_{2}$ can be genus embedded into the surface $S_{\gamma(s, k)-1}$. Then for each of the twelve possible values of $k$, i.e., $k=$ $0,1, \ldots, 11$, let $\Psi$ denote a genus embedding of $K_{m}$ into surface $S_{\gamma(s, k)}$ when $k \neq 2,5$ and a genus embedding of $K_{m}-K_{2}$ into surface $S_{\gamma(s, k)-1}$, when $k=2$ or 5 .

Then let $\Psi^{\prime}$ be a second copy of $K_{m}$ (respectively, $K_{m}-K_{2}$ when $k=2,5$ ) into a second copy of the surface $S_{\gamma(s, k)}$ (respectively, $S_{\gamma(s, k)-1}$ when $\left.k=2,5\right)$ in identically the same way. Now choose a value for $l, 1 \leq l \leq l_{k}(s)$. We proceed to choose $l$ faces of $\Psi$ and $l$ faces of $\Psi^{\prime}$ in a certain way and then to connect the two surfaces via $l$ "cylinders" or "tubes" joining the interiors of the faces chosen in $\Psi$ to the interiors of the faces chosen in $\Psi^{\prime}$.

If $k=2,5$, let $v_{0}$ be any vertex of $K_{m}-K_{2}$ which is not an endvertex of the missing edge. (If $k=2$ or 5 , it is known that $\Psi$ is a triangulation [R1] [RY1] [J1].) In this case let $v_{1}, \ldots, v_{m-1}$ denote the other vertices of $\Psi$ in clockwise order about the vertex $v_{0}$. Then for each $i=1, \ldots, m-2$, let $f_{i}$ be the face incident with $v_{0}$ which contains edges $v_{0} v_{i}$ and $v_{0} v_{i+1}$ in its boundary.

Fix a value for $\lambda, 1 \leq \lambda \leq l \leq l_{k}(s)$. We select $\lambda$ of the faces $f_{i}$ which are consecutive about $v_{0}$ and then every second face until we have chosen a total of $l$ faces in $\Psi$. In particular, we define our "chosen" faces $F_{i}$ by:

$$
F_{i}= \begin{cases}f_{i}, & 1 \leq i \leq \lambda \\ f_{2 i-\lambda}, & \lambda+1 \leq i \leq l\end{cases}
$$

Now we turn our attention to $\Psi^{\prime}$. In $\Psi^{\prime}$, denote the vertex corresponding to $v_{i}$ in $\Psi$ by $v_{i}^{\prime}$ for $i=0, \ldots, m-1$. In $\Psi^{\prime}$, starting from the face $F_{1}^{\prime}$, select every other face incident with $v_{0}^{\prime}$ and call these faces $F_{1}^{\prime}, \ldots, F_{l}^{\prime}$ (in any order). Name the two vertices on the boundary of $F_{i}^{\prime}$ that are adjacent to $v_{0}^{\prime}$ by $a_{i}^{\prime}$ and $b_{i}^{\prime}$, respectively.

For the cases when $k=1,6,9,10$, we shall proceed slightly differently. We know that the embedding is not a triangulation, so select a non-triangular face $F$ of $\Psi$ as $F_{1}$, and the corresponding face in $\Psi^{\prime}$ as $F_{1}^{\prime}$. Since face $F_{1}$ is not a triangle, we can choose four vertices $v_{0}, v_{1}, v, v_{2}$ on the boundary of $F_{1}$ such that edges $v_{0} v_{1}$ and $v_{0} v_{2}$ are adjacent boundary edges of $F_{1}$. We may name $v_{1}$ and $v_{2}$ such that as one traverses the boundary of $F_{1}$, one encounters the vertices $v_{1}, v$ and $v_{2}$ in clockwise order. In these 
cases when choosing the faces $F_{i}^{\prime}$ in $\Psi^{\prime}$ we should not choose any face with edge $v_{0}^{\prime} v^{\prime}$ on its boundary.

It is important to have this non-triangular face so that we may add an extra edge on the first tube in our construction. This additional edge will suffice to attain the bound in Lemma 3.1 below.

For the remaining cases when $k=0,3,4,7,8$ and 11 , choose vertices $v_{0}, v_{1}, \ldots, v_{m-1}$ as we did for the cases $k=2$ and 5 .

For each $i, 1 \leq i \leq l$, insert a tube $T_{i}$ joining the interiors of faces $F_{i}$ and $F_{i}^{\prime}$. We now add edges to form a new graph on $2 m$ vertices as follows. For $1 \leq i \leq \lambda$, add edges $v_{0} a_{i}^{\prime}, v_{0} b_{i}^{\prime}, v_{i} v_{0}^{\prime}, v_{i} a_{i}^{\prime}, v_{i} b_{i}^{\prime}, v_{i+1} a_{i}^{\prime}$ all on tube $T_{i}$. Also when $k=1,6,9,10$ add the "extra" edge $v_{0} v^{\prime}$ on tube $T_{1}$. When $\lambda+1 \leq i \leq l$, add edges $v_{0} a_{i}^{\prime}, v_{0} b_{i}^{\prime}, v_{2 i-\lambda} v_{0}^{\prime}, v_{2 i-\lambda} a_{i}^{\prime}, v_{2 i-\lambda} b_{i}^{\prime}$ and $v_{2 i-\lambda+1} a_{i}^{\prime}$ all on tube $T_{i}$. (See Figure 3.1.)

We will call the embedded graph on $2 m$ vertices constructed above $H_{I A}(s, k, l, \lambda)$. It is important to note that all edges added across the $l$ tubes in the above construction are distinct; i.e., graph $H_{I A}(s, k, l, \lambda)$ has no multiple edges. This is because all faces chosen in $\Psi^{\prime}$ only have $v_{0}^{\prime}$ on their common boundary, and hence all $a_{i}^{\prime}$ 's and $b_{i}^{\prime}$ 's are distinct. The parameter $\lambda$ which is the number of consecutive faces chosen in $\Psi$ determines the connectivity of the resulting graph.

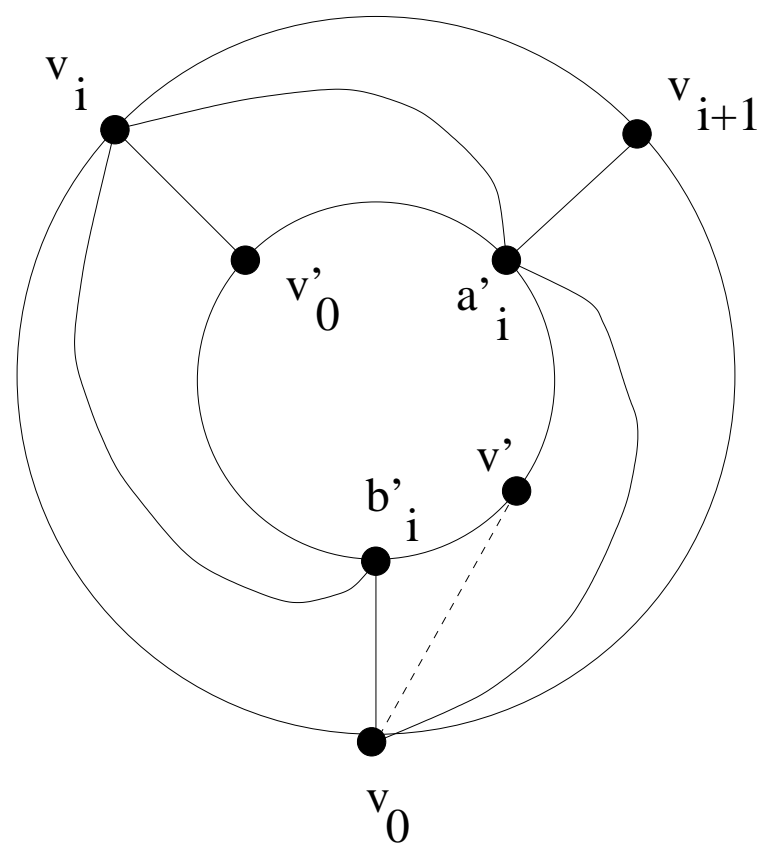

Figure 3.1(a) 


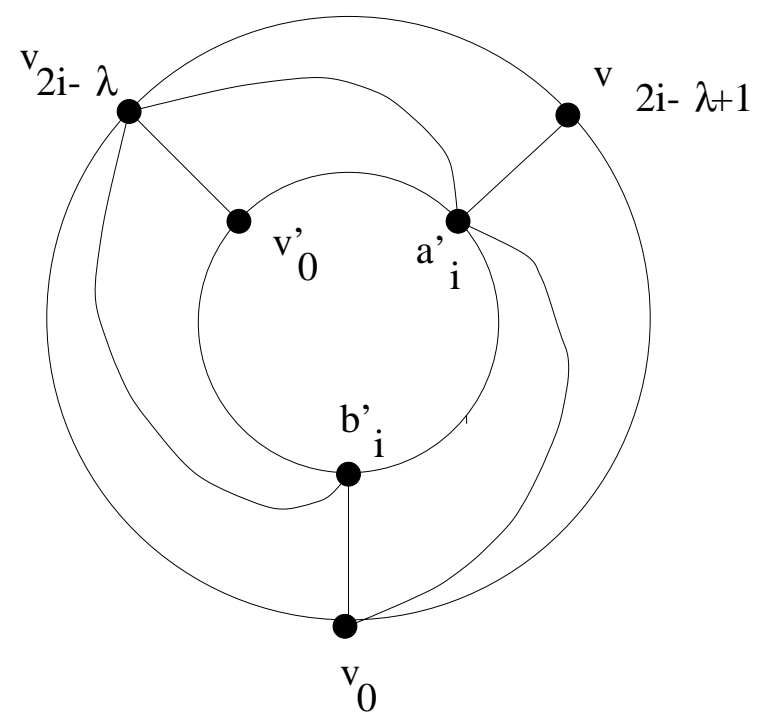

Figure 3.1(b)

We proceed to determine the genus and the connectivity of the graph $H_{I A}(s, k, l, \lambda)$. We will make use of the following corollary of Euler's formula (see [W1, pg. 62 and pg. $179])$.

Lemma 3.1. For all simple graphs $G$ with $p$ vertices and $q$ edges,

$$
\gamma(G) \geq \frac{q}{6}-\frac{p}{2}+1
$$

and

$$
\bar{\gamma}(G) \geq \frac{q}{3}-p+2 .
$$

Furthermore, equality holds in the above inequalities if and only if there is a triangular embedding of $G$ in its surface of minimum genus.

Now we compute the genus and connectivity of $H_{I A}(s, k, l, \lambda)$.

\section{Theorem 3.2.}

(i) The embedding of $H_{I A}(s, k, l, \lambda)$ constructed above is a genus embedding, and

$$
\gamma\left(H_{I A}(s, k, l, \lambda)\right)= \begin{cases}2 \gamma(s, k)+l-1, & \text { for } k \neq 2,5 \\ 2 \gamma(s, k)+l-3, & \text { for } k=2,5\end{cases}
$$

(ii) For all $s \geq 1, \kappa\left(H_{I A}(s, k, l, \lambda)\right)=2 l-\lambda+2$. 
Proof: First suppose that $k \neq 2$ or 5 . Denote $H_{I A}(s, k, l, \lambda)$ by $H$. Then $p_{H}=$ $2 m=2(12 s+k)$ and $q_{H}=(12 s+k)(12 s+k-1)+6 l+\epsilon_{k}$, where

$$
\epsilon_{k}= \begin{cases}0 & \text { if } k \neq 1,6,9,10 \\ 1 & \text { if } k=1,6,9,10\end{cases}
$$

Then by Lemma 3.1,

$$
\begin{aligned}
\gamma(H) & \geq\left\lceil\frac{q}{6}-\frac{p}{2}+1\right\rceil \\
& =\left\lceil\frac{1}{6}\left((12 s+k)(12 s+k-1)+6 l+\epsilon_{k}\right)-(12 s+k)+1\right\rceil \\
& =24 s^{2}+4 s k-14 s+l+\left\lceil\frac{k^{2}-7 k+6+\epsilon_{k}}{6}\right\rceil .
\end{aligned}
$$

Let us denote the right side of the final equation above by $A$.

On the other hand, it is known (cf. [R1]) that $\gamma\left(K_{12 s+k}\right)=12 s^{2}+2 s k-7 s+$ $\left\lceil\left(k^{2}-7 k+12\right) / 12\right\rceil$. Denote the right side of this equality by $B$. Then in each of the ten cases when $k \neq 2,5$, it is easy to check that $A=2 B+l-1$. But we know that the constructed surface is obtained by joining $l$ tubes between two surfaces each having genus $B$. Therefore the genus of the constructed surface is $2 B+l-1$.

Now suppose $k=2$ or 5 . Then $p_{H}=2(12 s+k)$ as before, but now

$$
\begin{aligned}
q_{H}=2((12 s+k)(12 s+k & -1) / 2-1)+6 l, \text { so } \\
\gamma(H) & \geq\left\lceil\frac{q}{6}-\frac{p}{2}+1\right\rceil \\
= & 24 s^{2}+4 s k-14 s+l+\left\lceil\frac{k^{2}-7 k+4}{6}\right\rceil .
\end{aligned}
$$

If we denote the right side of the last equality by $A^{\prime}$ then again it is easy to show that $A^{\prime}=2 B+l-3$ when $k=2$ and $k=5$. In these two cases the constructed surfaces are obtained by joining $l$ tubes between two surfaces each having genus $B-1$, and therefore the genus of the constructed surface is $2 B+l-3$. This proves (i).

Now we consider the connectivity of graph $H_{I A}(s, k, l, \lambda)$.

Since $1 \leq \lambda \leq l \leq l_{k}(s)$, it is easy to check that, for all $m=12 s+k$ and $s \geq 1$.

$$
2 l-\lambda+2 \leq \begin{cases}m-3, & \text { for } k \neq 2,5 \text { and } \\ m-4, & \text { for } k=2,5 .\end{cases}
$$

It now follows that given two vertices $u$ and $w$ both in $\Psi$, or both in $\Psi^{\prime}$, since when $k \neq 2,5, K_{m}$ is $(m-1)$-connected and when $n=2,5, K_{m}-K_{2}$ is $(m-2)$-connected, there must be more than $2 l-\lambda+2$ vertex-disjoint $u-w$ paths in $\left.H_{I A}(s, k, l, \lambda)\right)$. So we need only treat the remaining case, namely, when $u$ is a vertex in $\Psi$ and $w$ is a vertex in $\Psi^{\prime}$. 
Since

$$
M=\left\{v_{i} b_{i}^{\prime} \mid 1 \leq i \leq \lambda\right\} \cup\left\{v_{2 i-\lambda} b_{i}^{\prime} \mid \lambda+1 \leq i \leq l\right\} \cup\left\{v_{2 i-\lambda+1} a_{i}^{\prime} \mid \lambda \leq i \leq l\right\} \cup\left\{v_{0} a_{1}^{\prime}\right\} .
$$

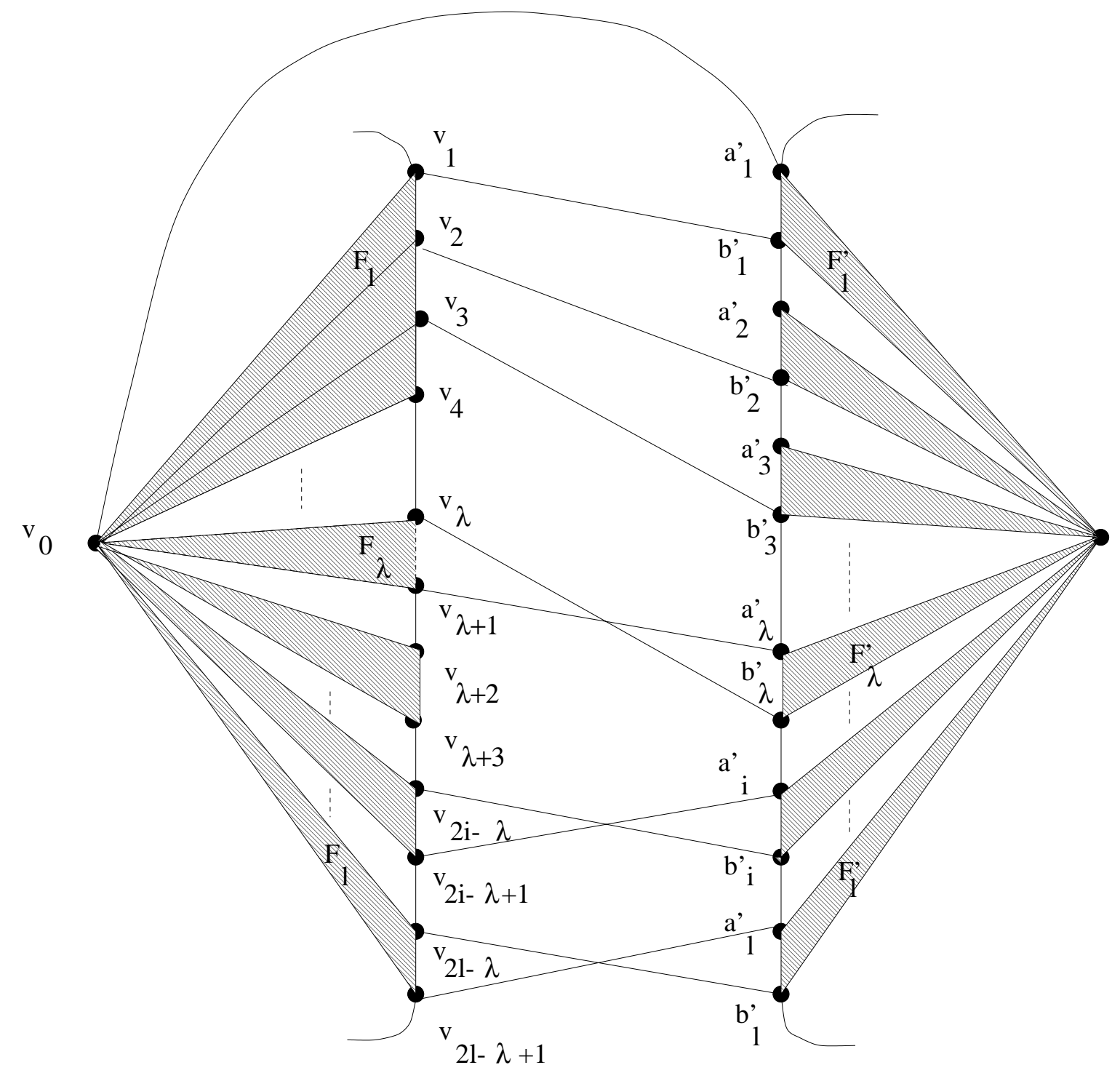

Figure 3.2

(See Figure 3.2.) is a matching of size $2 l-\lambda+2$ from vertices in $\Psi$ to vertices in $\Psi^{\prime}$, we have $2 l-\lambda+2$ vertex-disjoint paths, using these matching edges to join $u \in \Psi$ and $v \in \Psi^{\prime}$. Therefore $\kappa\left(H_{I A}(s, k, l, \lambda)\right) \geq 2 l-\lambda+2$. Since the set of all vertices of matching $M$ lying in $\Psi$ is a vertex cut, we have $\kappa\left(H_{I A}(s, k, l, \lambda)\right) \leq 2 l-\lambda+2$. Thus $\kappa\left(H_{I A}(s, k, l, \lambda)\right)=2 l-\lambda+2$, and the proof of the Theorem is complete. 
Note that $\kappa\left(H_{I A}(s, k, l, 1)\right)=2 l+1$ and for all $\lambda, 2 \leq \lambda \leq l, \kappa\left(H_{I A}(s, k, l, \lambda)\right) \leq$ $2 l+1$. For saturation purposes, the value of $\kappa\left(H_{I A}(s, k, l, 1)\right)$ is not quite high enough, so we extend our Construction IA to include, for all values of $k$, a graph $H_{I B}(s, k, l)$ having $\kappa=2 l+2$ and for $k=1,6,8,9,10$ and 11, a graph $H_{I C}(s, k, l)$ having $\kappa=2 l+3$.

To build graphs $H_{I B}(s, k, l)$ and $H_{I C}(s, k, l)$, we modify the Construction IA in the case $\lambda=1$ as follows. Let $\Psi$ be constructed just as before and let the faces $F_{1}=f_{1}, F_{2}=f_{3}, \ldots, F_{l-1}=f_{2 l-3}$ which meet only at vertex $v_{0}$ be as before. We proceed to modify the choice of face $F_{l}$ as follows. For graph $H_{I B}(s, k, l)$, let $F_{l}$ be the face formed by taking the two consecutive faces $f_{2 l-1}$ and $f_{2 l}$ and deleting their common boundary edge $v_{0} v_{2 l}$. (It is an easy calculation to show that since $s \geq 1$, there are enough faces at $v_{0}$ to make the above selection in such a way that faces $f_{2 l}$ and $f_{1}$ have no common boundary edge.)

We proceed to choose faces $F_{1}^{\prime}, \ldots, F_{l}^{\prime}$ in exactly the same way relative to embedding $\Psi^{\prime}$ as $F_{1}, \ldots, F_{l}$ were chosen relative to embedding $\Psi$.

Thus faces $F_{l}$ and $F_{l}^{\prime}$ will have at least four distinct vertices in their facial walks.

Now, as before, we insert tubes $T_{i}$ joining the interiors of $F_{i}$ and $F_{i}^{\prime}$, for $i=1, \ldots, l$. For each tube $T_{i}, i=1, \ldots, l-1$ we add edges across $T_{i}$ exactly as we did in Construction IA.

Recall that face $F_{l}$ is at least a quadrilateral and the four vertices $v_{0}, v_{2 l-1}, v_{2 l}$ and $v_{2 l+1}$ appear in clockwise order about the boundary of $F_{l}$. Similarly in the embedding $\Psi^{\prime}$, the vertices $v_{0}^{\prime}, v_{2 l-1}^{\prime}, v_{2 l}^{\prime}, v_{2 l+1}^{\prime}$ lie in counterclockwise order along the face boundary of $F_{l}^{\prime}$.

Across tube $T_{l}$ we insert the eight edges $v_{0} v_{2 l}^{\prime}, v_{0} v_{2 l+1}^{\prime}, v_{2 l-1} v_{2 l+1}^{\prime}$, $v_{2 l-1} v_{0}^{\prime}, v_{2 l} v_{0}^{\prime}, v_{2 l} v_{2 l-1}^{\prime}, v_{2 l+1} v_{2 l-1}^{\prime}, v_{2 l+1} v_{2 l}^{\prime}$. (See Figure 3.3.)

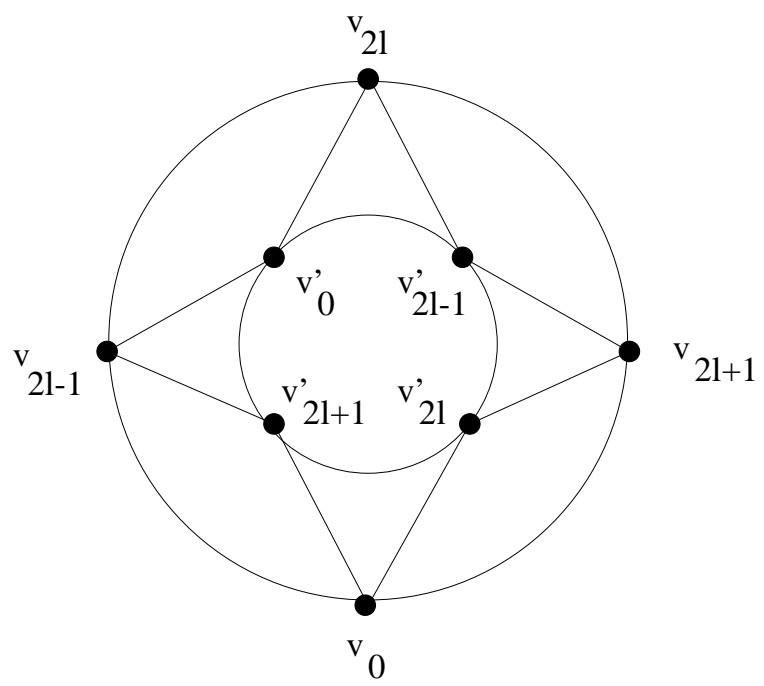

Figure 3.3

To construct graph $H_{I C}(s, k, l)$, for $k=1,6,8,9,10,11$, proceed just as in the 
construction of graph $H_{I B}(s, k, l)$ up through the selection of faces $F_{1}$ through $F_{l-1}$.

But this time let $F_{l}$ be the face formed by considering the three consecutive faces $f_{2 l-1}, f_{2 l}$ and $f_{2 l+1}$ and deleting the edges $v_{0} v_{2 l}$ and $v_{0} v_{2 l+1}$. (Again it is easy to show that for each value of $k, k \in\{1,6,8,9,10,11\}$, faces $f_{2 l+1}$ and $f_{1}$ have no common boundary edge.) Thus this time, $\Psi$ and $\Psi^{\prime}$ are both embeddings of $K_{m}$ with two adjacent edges removed. Thus the five vertices $v_{0}, v_{2 l-1}, v_{2 l}, v_{2 l+1}, v_{2 l+2}$ appear in clockwise order about the boundary of $F_{l}$.

Faces $F_{1}^{\prime}, \ldots, F_{l}^{\prime}$ are chosen similarly, except that $v_{0}^{\prime}, v_{2 l-1}^{\prime}, v_{2 l}^{\prime}, v_{2 l+1}^{\prime}$ and $v_{2 l+2}^{\prime}$ lie in counter-clockwise order along the face boundary of $F_{l}^{\prime}$.

Thus faces $F_{l}$ and $F_{l}^{\prime}$ will have at least five distinct vertices in their facial walks.

Add tubes $T_{1}, \ldots, T_{l}$ and six edges across each of $T_{1}, \ldots, T_{l-1}$ exactly as before. Next, across tube $T_{l}$ we insert the ten edges $v_{0} v_{2 l+1}^{\prime}, v_{0} v_{2 l+2}^{\prime}, v_{2 l-1} v_{2 l+2}^{\prime}, v_{2 l-1} v_{0}^{\prime}, v_{2 l} v_{0}^{\prime}$, $v_{2 l} v_{2 l-1}^{\prime}, v_{2 l+1} v_{2 l-1}^{\prime}, v_{2 l+1} v_{2 l}^{\prime}, v_{2 l+2} v_{2 l}^{\prime}, v_{2 l+2} v_{2 l+1}^{\prime}$.

\section{Theorem 3.3.}

(i) The above embeddings of $H_{I B}(s, k, l)$ and $H_{I C}(s, k, l)$ are genus embeddings, and

$$
\begin{aligned}
& \gamma\left(H_{I B}(s, k, l)\right)=\left\{\begin{array}{l}
2 \gamma(s, k)+l-1 \text { for } k \neq 2,5, \\
2 \gamma(s, k)+l-3 \text { for } k=2,5, \text { and }
\end{array}\right. \\
& \gamma\left(H_{I C}(s, k, l)\right)=2 \gamma(s, k)+l-1 \text { for } k=1,6,8,9,10 \text { and } 11 \text { and }
\end{aligned}
$$

(ii) $\kappa\left(H_{I B}(s, k, l)\right)=2 l+2$ and $\kappa\left(H_{I C}(s, k, l)\right)=2 l+3$.

Proof: The proof proceeds just as in the proof of Theorem 3.2. For Part (i), the only difference is that here for $H_{I B}(s, k, l)$ we have deleted one edge from each of $\Psi$ and $\Psi^{\prime}$, but have added two additional edges across tube $T_{1}$. Thus the total edge counts remain the same as in Theorem 3.2 in all cases. Similarly, for $H_{I C}(s, k, l)$, we have deleted two edges from each of $\Psi$ and $\Psi^{\prime}$, but have added four additional edges across $T_{1}$ and again the total edge counts remain the same as in Theorem 3.2 in all cases.

For Part (ii), the only difference is that, in $H_{I B}(s, k, l)$ and $H_{I C}(s, k, l)$, the size of the matching from $\Psi$ to $\Psi^{\prime}$ are increased by 1 and 2, respectively. This increases the connectivity of the graphs by 1 and 2 , respectively. 


\section{Construction II - the Orientable Case}

In this section we will construct graphs and orientable embeddings thereof such that the connectivities of these graphs will cover a higher range of values than that covered by the graphs and embeddings constructed in Section 3 .

As before, let $m=12 s+k$ and assume $s \geq 1$ and $0 \leq k \leq 11$. Let us once again begin by genus embedding graph $K_{m}$ into orientable surface $S_{\gamma(s, k)}$ when $k \neq 2,5$ and genus embedding graph $K_{m}-K_{2}$ into $S_{\gamma(s, k)-1}$ when $k=2$ or 5 . Call this embedding $\Psi_{0}$. Now take another identical embedding of the same graph in the same surface and call it $\Psi_{0}^{\prime}$.

Again this time we will first modify the underlying graphs of the two embeddings, and then proceed to link the two embeddings of the modified graphs with a collection of $l$ tubes. Once again we then insert new edges across these tubes so as to form one larger graph embedded in the composite surface.

Let us start with $k=2$ or 5 . Since $\Psi_{0}$ is an embedding of $K_{m}-K_{2}$, let $v_{0}$ denote any vertex not an endvertex of the missing edge. Remembering that $\Psi_{0}$ is a triangulation in these cases, let us label the vertices about vertex $v_{0}$ as $v_{1}, \ldots, v_{m-1}$ in clockwise order such that $v_{1}$ is also not an endvertex of the missing edge.

Label the vertices in $\Psi_{0}^{\prime}$ as $v_{0}^{\prime}, \ldots, v_{m-1}^{\prime}$ in exactly the same way as the vertices were labeled in $\Psi_{0}$. Therefore the labels appear in the clockwise order around $v_{0}$ and $v_{0}^{\prime}$, as indicated in Figure 4.1. This ordering is important to avoid multiple edges in the constructions.

Now suppose $k=0,3,4$ or 7 . Since $\Psi_{0}$ is a triangulation in these cases, choose any vertex and label it $v_{0}$. Then label the other $m-1$ vertices as $v_{1}, \ldots, v_{m-1}$ clockwise about $v_{0}$. Again do exactly the same labeling in $\Psi_{0}^{\prime}$.

For the remaining values of $k$, namely, $k=1,6,8,9,10$ and 11 , since $\Psi_{0}$ is not a triangulation, there must exist a face of embedding $\Psi_{0}$ which is of size at least 4 . Choose four vertices on the boundary of this face which are consecutive in a clockwise direction about this face and call them $v_{2}, v_{0}, v_{1}$ and $v$ respectively. It is important to have this non-triangular face. For each of these cases, the addition of two extra edges on one of the connecting tubes suffices to attain the genus bound in Lemma 3.1.

Now in all twelve cases (for each of the twelve values of $k$ ) delete vertex $v_{0}$ and denote the new face formed by $F_{0}$. Once again repeat the above labeling and subsequent vertex deletion in exactly the same way in $\Psi_{0}^{\prime}$ and label with $F_{0}^{\prime}$ that face which corresponds to $F_{0}$. Call the resulting embeddings $\Psi$ and $\Psi^{\prime}$ respectively. We now have a face with all other vertices in $\Psi\left(\Psi^{\prime}\right)$ on its boundary. This is a major difference from the constructions in Section 3. By inserting a tube between these two big faces and adding edges on this tube, the resulting graph has higher connectivity than that obtained using Construction I. When inserting edges across the various tubes, we need to take particular care to avoid multiple edges.

Insert a tube (or cylinder) $T_{0}$ with one end in the interior of face $F_{0}$ and the other end in the interior of face $F_{0}^{\prime}$.

Now suppose $l_{k}(s)$ is still defined as in Section 3 and suppose $1 \leq l \leq l_{k}(s)$. Here $l$ will denote the total number of connecting tubes we will insert between $\Psi$ and $\Psi^{\prime}$ and 
hence tube $T_{0}$ will be counted by $l$. We proceed to locate and insert the remaining $l-1$ tubes.

Let us choose $l-1$ faces $F_{1}, \ldots, F_{l-1}$ clockwise about vertex $v_{1}$ such that the boundary of $F_{i}$ contains the adjacent edges $v_{1} a_{i}$ and $v_{1} b_{i}$, vertices $v_{1}, a_{i}$ and $b_{i}$ are labeled in clockwise order about face $F_{i}$, and such that

(1) no two of the $F_{i}$ have a common boundary edge,

(2) if $k=1,6,8,9,10,11$, then for all $1 \leq i \leq l-1,\left\{a_{i}, b_{i}\right\} \cap\left\{v_{2}, v, v_{m-1}\right\}=\emptyset$, and

(3) if $k=0,2,3,4,5,7$, then for all $1 \leq i \leq l-1,\left\{a_{i}, b_{i}\right\} \cap\left\{v_{2}, v_{m-1}\right\}=\emptyset$.

(It is easy to check that for all twelve values of $k$, such a selection of $l-1$ faces $F_{i}$ is possible, since $s \geq 1$.) Note that these $a_{i}$ 's and $b_{i}$ 's are neighbor vertices of $v_{1}$, and they are labeled as $v_{i}$ 's on the face boundary of $F_{0}$.

Again, make precisely the same selection of faces in $\Psi^{\prime}$. Then, for each $i=1, \ldots, l-$ 1 , insert a tube $T_{i}$ joining the interiors of faces $F_{i}$ and $F_{i}^{\prime}$. Then for each $i, 1 \leq i \leq l-1$, across tube $T_{i}$ add the six edges $v_{1} a_{i}^{\prime}, v_{1} b_{i}^{\prime}, a_{i} a_{i}^{\prime}, a_{i} v_{1}^{\prime}, b_{i} v_{1}^{\prime}, b_{i} b_{i}^{\prime}$.

We now proceed to add edges across tube $T_{0}$ as follows. Suppose $\alpha$ is an integer variable such that $3 \leq \alpha \leq m-2$. Consider the edge set $\left\{v_{1} v_{1}^{\prime}, v_{1} v_{2}^{\prime}, v_{2} v_{1}^{\prime}, v_{2} v_{m-1}^{\prime}, v_{2} v_{m-2}^{\prime}\right\} \cup$ $\left\{v_{i} v_{m-i+1}^{\prime} \mid 3 \leq i \leq \alpha-1\right\} \cup\left\{v_{i} v_{m-i}^{\prime} \mid 3 \leq i \leq \alpha-1\right\} \cup\left\{v_{\alpha} v_{j}^{\prime} \mid 2 \leq j \leq m-\alpha+1\right\} \cup\left\{v_{i} v_{2}^{\prime} \mid \alpha \leq\right.$ $i \leq m-1\}$. In the cases when $k=1,6,8,9,10,11$ we add to this set the two additional edges $v_{1} v^{\prime}$ and $v v_{1}^{\prime}$.

Finally, if $\alpha<\lfloor(m+1) / 2\rfloor$ and if $v_{\alpha}$ is one of the $a_{i}$ 's or $b_{i}$ 's, then we do not add edge $v_{\alpha} v_{\alpha}^{\prime}$ across tube $T_{0}$ because it already exists on one of the tubes $T_{1}, \ldots, T_{l-1}$. Similarly, if $\alpha \geq\lfloor(m+1) / 2\rfloor$ and if $v_{\lfloor(m+1) / 2\rfloor}$ is one of the $a_{i}$ 's or one of the $b_{i}$ 's, then we do not add edge $v_{\lfloor(m+1) / 2\rfloor} v_{\lfloor(m+1) / 2\rfloor}^{\prime}$ across tube $T_{0}$ as it already exists on one of $T_{1}, \ldots, T_{l-1}$. (See Figure 4.1.)

Call the resulting graph on $2 m-2$ vertices $H_{I I}(s, k, l, \alpha)$.

Lemma 4.1. Each graph $H_{I I}(s, k, l, \alpha)$ is simple.

Proof: On tube $T_{0}$, the edges incident with $v_{1}$ and $v_{1}^{\prime}$ are $v_{1} v_{1}^{\prime}, v_{1} v_{2}^{\prime}, v_{2} v_{1}^{\prime}$ and (possibly) $v v_{1}^{\prime}$ and $v_{1} v^{\prime}$. On the other hand, on tube $T_{i}$ the edges incident with $v_{1}$ and $v_{1}^{\prime}$ are $v_{1} a_{i}^{\prime}, v_{1} b_{i}^{\prime}, a_{i} b_{i}^{\prime} a_{i} v_{1}^{\prime}, b_{i} v_{1}^{\prime}$. But $\left\{a_{i}, b_{i}\right\} \cap\left\{v_{2}, v, v_{m-1}\right\}=\emptyset$ and and $\left\{a_{i}^{\prime}, b_{i}^{\prime}\right\} \cap$ $\left\{v_{2}^{\prime}, v^{\prime}, v_{m-1}^{\prime}\right\}=\emptyset$, so none of these edges is multiple.

The remaining edges on $T_{i}$ are of the form $a_{i} a_{i}^{\prime}$ or $b_{i} b_{i}^{\prime}$. But on $T_{0}$ we have constructed no such edges, because of the reversed order of $v_{i}^{\prime}$ 's and $v_{i}^{\prime}$ 's.

Let us now compute the genus of $H_{I I}(s, k, l, \alpha)$. 


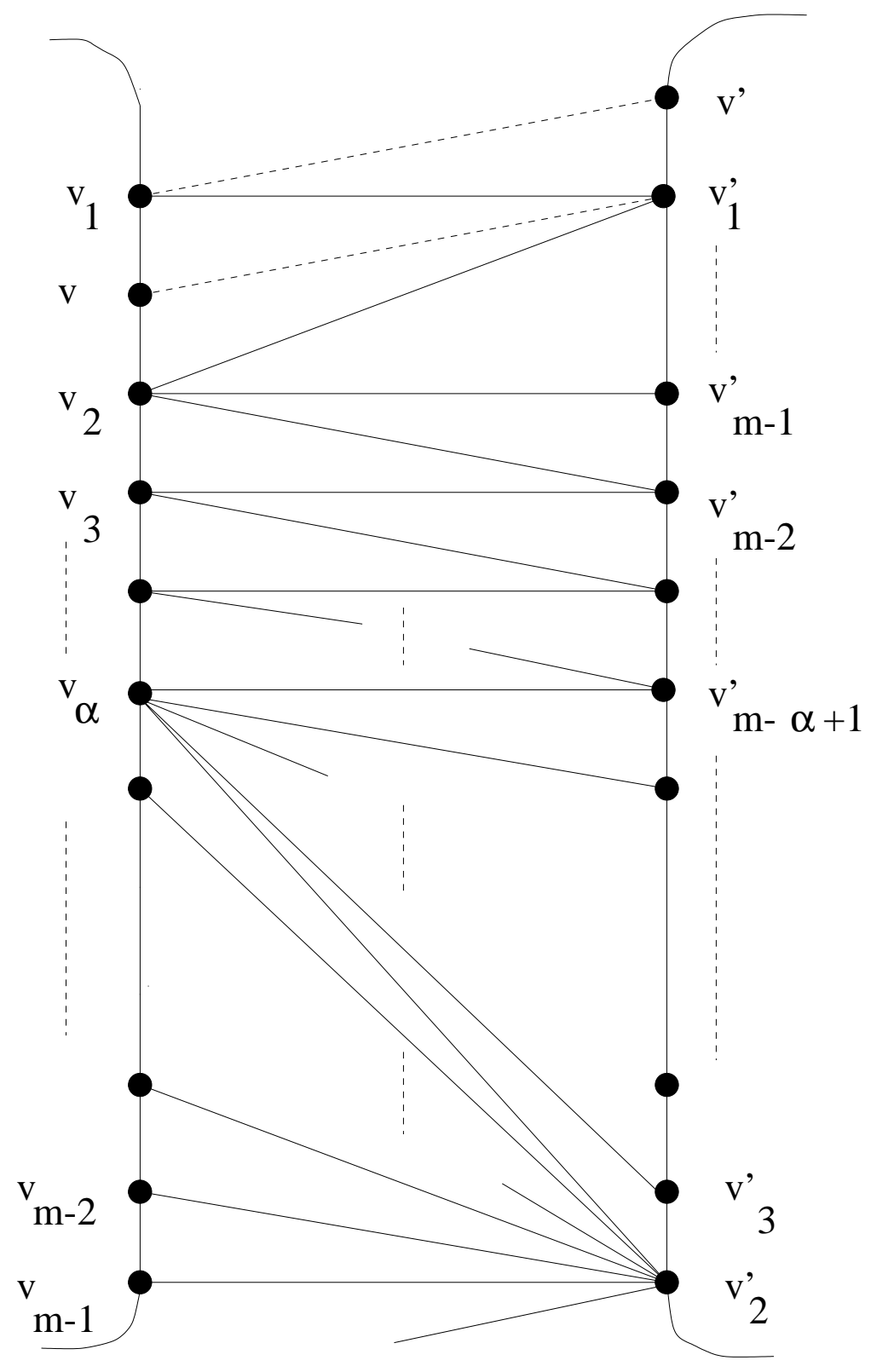

Figure 4.1

Theorem 4.2. The embedding of $H_{I I}(s, k, l, \alpha)$ constructed above is a genus embedding and

$$
\gamma\left(H_{I I}(s, k, l, \alpha)\right)= \begin{cases}2 \gamma(s, k)+l-3, & \text { if } k=2,5 \\ 2 \gamma(s, k)+l-1, & \text { if } k \neq 2,5 .\end{cases}
$$


Proof: For each $k=0, \ldots, 11$, we have embedded $H_{I I}(s, k, l, \alpha)$ in a surface with the required genus. Now noting that $q \geq 2[m(m-1) / 2-(m-1)-1]+6(l-1)+2(m-1)$, when $k=2,5 ; q \geq 2[(m-1)(m-2) / 2+2(m-1)+6(l-1)-1$, when $k=0,3,4$ or 7 ; and $q \geq 2(m-1)(m-2) / 2+2(m-1)+2+6(l-1)-1$, when $k=1,6,8,9,10$ or 11; and that $p=2(m-1)$ in all twelve cases, by Lemma 3.1 and an easy counting exercise we obtain

$$
\gamma\left(H_{I I}(s, k, l, \alpha)\right) \geq \begin{cases}2 \gamma(s, k)+l-3, & \text { if } k=2,5 \\ 2 \gamma(s, k)+l-1, & \text { if } k \neq 2,5,\end{cases}
$$

and the theorem is proved.

Now consider the connectivity of graph $H_{I I}(s, k, l, \alpha)$. Since we have two independent parameters $\alpha$ and $l$, in general it is impossible to determine when $v_{\alpha}$ is one of the $a_{i}$ 's or one of the $b_{i}$ 's. Moreover, the edges on the tubes $T_{i}, i \geq 1$, may affect the connectivity of the graphs. So it is not easy to find the exact value of $\kappa$. However, as we shall see below, the exact value will not be required.

To this end, it will be convenient to introduce some new terminology. Let any matching which matches vertices of $\Psi$ with vertices of $\Psi^{\prime}$ be called a left-right matching (or $L R$-matching). Let $\nu_{L R}\left(H_{I I}(s, k, l, \alpha)\right)$ denote the size of any largest $L R$ matching in $H_{I I}(s, k, l, \alpha)$.

\section{Theorem 4.3.}

$$
\kappa\left(H_{I I}(s, k, l, \alpha)\right) \geq \begin{cases}\nu_{L R}(H(s, k, l, \alpha)) & \text { if } k \neq 2,5 \\ \min \left\{\nu_{L R}(H(s, k, l, \alpha)), m-2\right\} & \text { if } k=2,5 .\end{cases}
$$

Proof: Denote $H_{I I}(s, k, l, \alpha)$ by $H$. Suppose $u$ and $w$ are two vertices of $H$. We want to consider the maximum number of vertex-disjoint paths between $u$ and $w$.

First suppose $k \neq 2,5$. If $u$ and $w$ are both in $\Psi$, then there exist $m-2$ disjoint paths joining them in $\Psi$ since $\Psi$ is an embedding of $K_{m-1}$. It is easy to construct one additional path joining $u$ and $w$ by using vertices in $\Psi^{\prime}$. Similarly, for $u$ and $w$ both in $\Psi^{\prime}$, it is easy to construct $m-1$ disjoint paths joining them.

So it will suffice to consider the case with $u$ is in $\Psi$ and $w$ is in $\Psi^{\prime}$.

Let $M_{\alpha}$ be a maximum $L R$-matching and suppose that the endvertices of $M_{\alpha}$ in $\Psi$ are $x_{1}, \ldots, x_{j}, j=\nu_{L R}$. Now $j \leq m-1$, so since $\Psi$ is an embedding of $K_{m-1}$, there exist $j \leq m-1$ disjoint paths from $u$ to each of the vertices $x_{1}, \ldots, x_{j}$. These paths are all single edges, unless $u \in\left\{x_{1}, \ldots, x_{j}\right\}$ and in that case one of the paths is of length 0 . Similarly there exist $j$ disjoint paths from $w$ to the endvertices of $M_{\alpha}$ in $\Psi^{\prime}$. Thus there are $j=\nu_{L R}$ disjoint $u-v$ paths in $H$. Hence $\kappa(H) \geq \nu_{L R}$ as claimed.

Now suppose $k=2$ or 5 . Then, if $\nu_{L R} \leq m-2$, proceeding exactly as in the case for $k \neq 2,5$, it may be shown that there are at least $\nu_{L R}$ disjoint paths joining $u$ and $w$. Finally, if $\nu_{L R}>m-2$, that is, if $\nu_{L R}=m-1$, then $\alpha=m-2$ and once again it is 
easy to find $m-2$ disjoint paths joining every two vertices $u$ and $w$ in $H$. The theorem follows.

If we set $\alpha=m-2$ in the above theorem, we obtain the next result.

\section{Corollary 4.4 .}

$$
\kappa\left(H_{I I}(s, k, l, m-2)\right) \begin{cases}=m-1 & \text { when } k \neq 2,5 \text { and } \\ \geq m-2 & \text { when } k=2,5 .\end{cases}
$$

Proof: Equality follows in the case $k \neq 2,5$ since $\operatorname{deg}\left(v_{m-1}^{\prime}\right)=m-1$.

The next result shows that if one increases parameter $\alpha$ by 1 , the corresponding connectivity of $H_{I I}(s, k, l, \alpha)$ changes by at most 1 .

Lemma 4.5. If $3 \leq \alpha \leq m-3$, then

$$
\kappa\left(H_{I I}(s, k, l, \alpha)\right)-1 \leq \kappa\left(H_{I I}(s, k, l, \alpha+1)\right) \leq \kappa\left(H_{I I}(s, k, l, \alpha)\right)+1 .
$$

Proof: Denote $H_{I I}(s, k, l, \alpha)$ by $H_{I I}(\alpha)$. Note first that $H_{I I}(\alpha)-v_{\alpha}$ is a spanning subgraph of $H_{I I}(\alpha+1)-v_{\alpha}$, so $\kappa\left(H_{I I}(\alpha)\right)-1 \leq \kappa\left(H_{I I}(\alpha)-v_{\alpha}\right) \leq \kappa\left(H_{I I}(\alpha+1)-v_{\alpha}\right) \leq$ $\kappa\left(H_{I I}(\alpha+1)\right)$ and the first inequality is proved.

Next note that $H_{I I}(\alpha+1)-v_{\alpha+1}$ is a spanning subgraph of $H_{I I}(\alpha)-v_{\alpha+1}$ and so $\kappa\left(H_{I I}(\alpha+1)\right)-1 \leq \kappa\left(H_{I I}(\alpha+1)-v_{\alpha+1}\right) \leq \kappa\left(H_{I I}(\alpha)-v_{\alpha+1}\right.$ and so $\kappa\left(H_{I I}(\alpha+1)\right) \leq$ $\kappa\left(H_{I I}(\alpha)-v_{\alpha+1}\right)+1 \leq \kappa\left(H_{I I}(\alpha)\right)+1$ and the second inequality is verified.

Now the proof of the following theorem is immediate.

Theorem 4.6. Suppose $a=\kappa\left(H_{I I}(s, k, l, 3)\right)$ and

$$
b= \begin{cases}m-1, & \text { if } k \neq 2,5 \text { and } \\ m-2, & \text { if } k=2,5 .\end{cases}
$$

Then for any $\kappa_{0} \in[a, b]$ there is an $\alpha \in[3, m-3]$ such that $\kappa\left(H_{I I}(s, k, l, \alpha)\right)=\kappa_{0}$.

Theorem 4.6 tells us the value of $b$ in the interval of connectivities $[a, b]$ which we can saturate. The next result gives us a useful upper bound on the other endpoint $a$.

\section{Theorem 4.7.}

$$
\kappa\left(H_{I I}(s, k, l, 3)\right) \leq \begin{cases}2 l+3 & \text { when } k=1,6,8,9,10 \text { or } 11 \text { and } \\ 2 l+2 & \text { when } k=0,2,3,4,5,7 .\end{cases}
$$


Proof: The set

$$
S_{0}= \begin{cases}\left\{v_{1}, v_{2}, v_{3}, v_{2}^{\prime}, v\right\}, & \text { when } k=1,6,8,9,10,11, \text { and } \\ \left\{v_{1}, v_{2}, v_{3}, v_{2}^{\prime}\right\}, & \text { when } k=0,2,3,4,5,7,\end{cases}
$$

covers all edges crossing tube $T_{0}$ and hence the set $S=S_{0} \cup\left\{a_{1}, b_{1}, \ldots, a_{l-1}, b_{l-1}\right\}$ covers all edges crossing any of the total of $l$ tubes. So set $S$ is a cutset separating vertex $v_{m-1}$, say, from the remaining vertices of $\Psi^{\prime}$ and has the size claimed in the statement of the theorem.

Now we turn our attention to the high end of the connectivity interval $[a, b]$ discussed above. By Theorem 4.6 we know that for each $l$ we can saturate $[2 l+3, m-1]$, when $k=1,6,8,9,10,11$, over $[2 l+2, m-1]$ when $k=0,3,4,7$, and over $[2 l+2, m-2]$ when $k=2,5$. For saturation reasons we want to construct graphs with genus $=$ $\gamma\left(H_{I I}(s, k, l, \alpha)\right)$ and built from $H_{I I}(s, k, l, \alpha)$ by modifying the arrangement of edges on tube $T_{0}$ so that the resulting graphs have connectivities $m-1$ when $k=2,5$ and $m$ when $k \neq 2,5$.

If $k=0,3,4$ or 7 , begin just as in the case of $H_{I I}(s, k, l, m-2)$ as far as selecting faces and placing tubes is concerned. Suppose that $k=1,6,7,8,9,10$ or 11 . In locating tubes $T_{1}, \ldots, T_{l-1}$, we modify our previous choices for the $a_{i}$ 's and $b_{i}$ 's so that $\left\{a_{i}, b_{i}\right\} \cap$ $\left\{v_{2}, v_{m-1}, v\right\}=\emptyset$.

Suppose, then, that $k=2$ or 5 . Recall that we began the construction in this case by embedding a copy of $K_{m}-K_{2}$ and then deleted vertex $v_{0}$ which was not a vertex of the missing edge. Denote this missing edge by $x y$. Let us now choose our $l-1$ pairs $\left\{a_{i}, b_{i}\right\}$ so that $\left\{a_{i}, b_{i}\right\} \cap\left\{v_{2}, v_{m-1}, x\right\}=\emptyset$.

For $k=0,3,4,7$ and for $k=2,5$, modify the graph $H_{I I}(s, k, l, m-2)$ by replacing edges $v_{1} v_{2}^{\prime}$ and $v_{2} v_{1}^{\prime}$ with edges $v_{1} v_{m-1}^{\prime}$ and $v_{m-1} v_{1}^{\prime}$. When $k=1,6,8,9,10,11$, modify $H_{I I}(s, k, l, m-2)$ by replacing edges $v_{1} v_{2}^{\prime}, v_{1} v^{\prime}, v v_{1}^{\prime}$ and $v_{2} v_{1}^{\prime}$ with edges $v_{1} v_{m-1}^{\prime}, v v_{m-1}^{\prime}$, $v_{m-1} v^{\prime}$ and $v_{m-1} v_{1}^{\prime}$. In each case, denote the graph obtained by $H_{I}(k, l, m)$.

\section{Theorem 4.8.}

$$
\kappa\left(H_{I}(k, l, m)\right)= \begin{cases}m, & \text { if } k \neq 2,5 \text { and } \\ m-1, & \text { if } k=2,5\end{cases}
$$

Proof: Among the edges across tube $T_{0}$ call $v_{1} v_{1}^{\prime}, v_{2} v_{m-1}^{\prime}, v_{3} v_{m-2}^{\prime}, \ldots, v_{m-1} v_{2}^{\prime}$, the horizontal matching, and call $v_{1} v_{m-1}^{\prime}, v_{2} v_{m-2}^{\prime}, v_{3} v_{m-3}^{\prime}, \ldots, v_{m-2} v_{2}^{\prime}, v_{m-1} v_{1}^{\prime}$ the slanted matching. (It is important to realize that the edge $v_{\lfloor(m+1) / 2\rfloor} v_{\lfloor(m+1) / 2\rfloor}^{\prime}$ will be present, whether it is drawn on one of the tubes $T_{1}, \ldots, T_{l-1}$ or on the tube $T_{0}$.)

First, suppose $k=0,3,4$ or 7 .

Let $u$ and $w$ be two vertices of $H_{I}(k, l, m)$. If both vertices lie in $\Psi$, then it is easy to find $m$ vertex-disjoint paths joining them, where two of these paths use vertices of $\Psi^{\prime}$. By symmetry, if both lie in $\Psi^{\prime}$, there are $m$ vertex-disjoint paths joining them as well. 
Now suppose $u$ lies in $\Psi$ and $w$ lies in $\Psi^{\prime}$; say, without loss of generality that $u=v_{i}$ and $w=v_{j}^{\prime}$.

(i) First suppose $j \leq m-i$. There are three subcases.

(i-1) Suppose $j=m-i$, that is, suppose edge $v_{i} v_{j}^{\prime}$ is an edge of the slanted matching.

Then there are $m-3 u-w$ paths of length 3 , using the $m-3$ horizontal matching edges incident with $v_{1}, \ldots, v_{i-1}, v_{i+2}, \ldots, v_{m-1}$. To these we add the three additional $u-w$ paths $v_{i} v_{j+1}^{\prime} v_{j}^{\prime}, v_{i} v_{j}^{\prime}, v_{i} v_{i+1} v_{j}^{\prime}$ to obtain a total of $m$ vertex-disjoint $u-w$ paths.

(i-2) Suppose $j=m-i-1$. There are $m-4 u-w$ paths of length 3 , using the $m-4$ horizontal matching edges incident with $v_{1}, \ldots, v_{i-1}, v_{i+3}, \ldots, v_{m-1}$. To these we add the four additional $u-w$ paths $v_{i} v_{j+2}^{\prime} v_{j}^{\prime}, v_{i} v_{j+1}^{\prime} v_{j}^{\prime}, v_{i} v_{i+1} v_{j}^{\prime}$ and $v_{i} v_{i+2} v_{j}^{\prime}$, to obtain our $m$ vertex-disjoint $u-w$ paths.

(i-3) Suppose $j \leq m-i-2$; say, $j=m-t$, for some $t \geq i+2$. In this case, there are $m-4 u-w$ paths of length 3 , using the horizontal matching edges incident with vertices $v_{1}, \ldots, v_{i-1}, v_{t+2}, v_{t+3}, \ldots, v_{m-1}$ together with the slanted matching edges incident with vertices $v_{i+1}, \ldots, v_{t-1}$. To these paths we add the additional four paths of length 2: $v_{i} v_{m-i+1}^{\prime} v_{j}^{\prime}, v_{i} v_{m-i}^{\prime} v_{j}^{\prime}, v_{i} v_{t}^{\prime} v_{j}^{\prime}$ and $v_{i} v_{t+1} v_{j}^{\prime}$.

(ii) Next, suppose $j=m-i+1$.

In this case we use the $m-3$ slanted matching edges incident with the $m-3$ vertices $v_{1}, v_{2}, \ldots, v_{i-2}, v_{i+1}, \ldots, v_{m-1}$, together with the three paths $v_{i} v_{i-1} v_{j}^{\prime}, v_{i} v_{j}^{\prime}$ and $v_{i} v_{j+1}^{\prime} v_{j}^{\prime}$ to get $m$ vertex-disjoint $u-w$ paths.

(iii) Finally, suppose $j>m-i+1$.

This is the same as Case (i), if one performs the same cyclic relabeling on both sides. claimed.

On the other hand, however, $\operatorname{deg} H_{I}(k, l, m)\left(v_{m-1}\right)=m$, so $\kappa\left(H_{I}(k, l, m)\right)=m$ as

It then follows that $\kappa\left(H_{I}(k, l, m)\right)=m$ in the cases $k=1,6,8,9,10$ and 11 , for observe that the "extra vertex" $v$ is just one of the vertices $v_{1}, \ldots, v_{m-1}$ appearing at a different place on the facial walk of the face produced by the deletion of vertex $v_{0}$.

Next, suppose $k=2$ or 5 . We need to show that $\kappa\left(H_{I}(k, l, m)\right)=m-1$. Notice that, when $k=2$ or 5 , as opposed to the cases when $k \neq 2$ or 5 , there is only one edge missing in each of $\Psi$ and $\Psi^{\prime}$, and these two missing edges are on different sides of the $L R$-matching on tube $T_{0}$. Since $\kappa\left(H_{I}(k, l, m)\right)=m$ for $k \neq 2,5$, by a similar argument, it is not hard to check that $\kappa\left(H_{I}(k, l, m)\right)=m-1$ for $k=2,5$.

Theorem 4.9. The embedding of $H_{I}(k, l, m)$ constructed above is a genus embedding and

$$
\gamma\left(H_{I}(k, l, m)\right)= \begin{cases}2 \gamma(s, k)+l-3, & \text { if } k=2,5 \\ 2 \gamma(s, k)+l-1, & \text { if } k \neq 2,5\end{cases}
$$

Proof: The proof is very similar to that of Theorem 4.2 . 


\section{The Interval of Saturation-the Orientable Case}

Before presenting the main theorem of this section, it is necessary to have the following results for surfaces of small orientable genus.

Lemma 5.1. Let $g$ be an integer such that $0 \leq g \leq 9$. Then for every integer $i \in\left[1, \kappa_{\max }\left(S_{g}\right)\right]$, there is a graph $G$ with $g=\gamma(G)$ and $\kappa(G)=i$.

For $g=10,11$, and every integer $i \in\left[1,(\sqrt{2} / 2) \kappa_{\max }\left(S_{g}\right)\right]$, there is a graph $G$ with $g=\gamma(G)$ and $\kappa(G)=i$.

Proof: When $g=0$, it is well known that there are planar graphs having vertex connectivity $5,4,3,2$ and 1 .

Secondly, we claim that it follows from the results of Negami [N1] that for every surface with orientable genus $\geq 1$ (or non-orientable genus $\geq 2$ ), there are graphs of that genus which have connectivity 6 , have a vertex of degree 6 , and which triangulate the surface. To see this in the orientable case, one need only start Negami's construction with a 6-regular 6-connected graph which triangulates the torus and has sufficiently many vertices so that not all vertices of the beginning graph are subsequently affected by Negami's construction. Similarly, if one begins with a 6-connected 6-regular triangulation of the Klein bottle which has sufficiently many vertices, at least one vertex of degree 6 will remain unmodified by the Negami construction.

Moreover, since the Negami graphs are all triangulations, one may remove up to five edges incident with a vertex of degree 6 without lowering the genus. Therefore we have the following proposition.

Proposition: Starting with a 6-connected triangulation of genus $\gamma \geq 1$ (or genus $\bar{\gamma} \geq 2$ ) which has a vertex of degree 6 , there are graphs with connectivity $5,4,3,2$ and 1 all of which have genus $\gamma$ (or $\bar{\gamma}$ ).

First consider the (orientable) genera 2 and 3. Since $\gamma\left(K_{8}\right)=2, \kappa\left(K_{8}\right)=7=$ $\kappa\left(S_{2}\right), \gamma\left(K_{9}\right)=\gamma\left(K_{9}-K_{2}\right)=3, \kappa\left(K_{9}\right)=8=\kappa_{\max }\left(S_{3}\right), \kappa\left(K_{9}-K_{2}\right)=7$, the lemma is true for $\gamma=2$ and 3.

Next consider the case when $\gamma=4$. Recall that $\gamma\left(K_{10}\right)=4$. Again by edge counting it is easily seen that the genus of each of $K_{10}-K_{2}$ and $K_{10}-2 K_{2}$ (where $2 K_{2}$ denotes two edges having a common vertex) is also 4 . So there are genus 4 graphs having connectivities $1, \ldots, 9$, where $\kappa_{g e n}\left(S_{4}\right)=\kappa_{\max }\left(S_{4}\right)=9$.

Similarly, there are graphs with $\kappa=1, \ldots, 10$ having $\gamma=5$ and $\kappa_{g e n}\left(S_{5}\right)=$ $\kappa_{\max }\left(S_{5}\right)$

$=10$ as well as graphs with $\kappa=1, \ldots, 11$ having $\gamma=6$ where $\kappa_{g e n}\left(S_{6}\right)=\kappa_{\max }\left(S_{6}\right)=$ 11.

For $S_{7}$ our approach is somewhat different. Embed $K_{9}$ and $K_{10}$, choose a vertex $v_{0}$ from $K_{9}$ and a vertex $v_{0}^{\prime}$ from the $K_{10}$, delete each, and insert cylinder $T_{0}$, just as in Construction II (see Section 4). (We do not add any additional tubes this time.) Insert the following edges: $v_{i} v_{i}^{\prime}$ and $v_{i} v_{i+1}^{\prime}$ for $i=1, \ldots, 8 ; v_{1} v^{\prime}$ and $v_{8} v_{1}^{\prime}$. It is then easy to check that the resulting graph is 9-connected and a simple edge count, together with Lemma 3.1, shows that the genus of this graph is 7 as desired. Modify this embedding 
by inserting different edges on tube $T_{0}$ can produce graphs with $\kappa=7$ and 8 with genus 7.

Consider next $S_{8}$. The octahedron $K_{7(2)}$, obtained from the complete graph $K_{14}$ by deleting a perfect matching has connectivity 12 and by [JR1,AG1], genus 8 . If an arbitrary vertex is selected and up to five edges incident with this vertex are removed, an edge count shows that each of the resulting graphs still has genus 8 . In this way graphs having connectivity $7, \ldots, 11$ are created. Thus the full connectivty interva for the surface $S_{8}$ can be saturated.

The graph $K_{14}-K_{2}$ triangulates the surface $S_{9}$ by [RY1] and [J1] and has connectivity 12. Again by a simple edge count it is easy to show that up to five edges can be deleted from some fixed vertex of this graph without lowering the genus of the resulting graph. So we can get genus 9 graphs having connectivity $1, \ldots, 12$ and thus can saturate the full connectivity interval for the surface $S_{9}$.

For surface $S_{10}$ we return to a modification of Construction II. Embed two copies of $K_{11}$, delete a vertex from each and construct the tube $T_{0}$ just as in Construction II. Across $T_{0}$ construct edges $v_{i} v_{i}^{\prime}$ and $v_{i} v_{i+1}^{\prime}$ for $i=1, \ldots, 9$ as well as edges $v_{10} v_{10}^{\prime}$ and $v_{10} v_{1}^{\prime}$. The resulting graph has $\kappa=11$ and an edge count verifies that the genus is indeed 10. A modification of the edge construction across $T_{0}$ similar to that done above for the genus 7 case yields genus 10 graphs having connectivity $1, \ldots, 11$ and since $(\sqrt{2} / 2) \kappa_{\max }\left(S_{10}\right)=9$, we are done. Surface $S_{11}$ is handled in much the same way as $S_{10}$. Embed $K_{11}$ in $S_{5}, K_{12}$ in $S_{6}$, delete a vertex from each and add tube $T_{0}$ as well as edges across $T_{0}$ as before. As before, graphs having connectivity $1, \ldots, 11$ can be constructed all of which have genus 11. But $(\sqrt{2} / 2) \kappa_{\max }\left(S_{11}\right)=9$ and again we are done.

We now need to construct genus embeddings whose connectivity cover the lower range of values in connectivity intervals. In doing so, we will make use of a graph operation which will be called splitting. Suppose graph $G$ is $n$-connected, where $n \geq 2$, and suppose $v \in V(G)$ with $\operatorname{deg} v=n$. Partition the neighborhood $N_{G}(v)$ into $A \cup B$ where $A \cap B=\emptyset$ and $|A| \geq 1$ and $|B| \geq 1$. Form a new graph $G^{\prime}$ from $G$ by deleting vertex $v$, inserting two new vertices $v_{1}$ and $v_{2}$ such that $v_{1}$ is adjacent to each vertex in $A$, vertex $v_{2}$ is adjacent to each vertex in $B$ and $v_{1}$ is adjacent to $v_{2}$. Suppose $|A|=a$ and $|B|=b$. We then say that graph $G^{\prime}$ is obtained from graph $G$ by an $(a, b)$-split at vertex $v$.

Lemma 5.2. Let $G$ be a graph with $\kappa(G) \geq 2$ and let $v$ be a vertex in $G$. Let $G^{\prime}$ be a graph obtained from graph $G$ by an $(a, b)$-split at vertex $v$ and suppose $r=\min \{1+a, 1+b\} \leq \kappa(G)$. Then $\kappa\left(G^{\prime}\right)=r$.

Proof. Note that $\left|N_{G^{\prime}}\left(v_{i}\right)\right| \geq r$, for $i=1,2$.

Now let $S^{\prime}$ be a minimum vertex cutset in $G^{\prime}$ and suppose $\left|S^{\prime}\right|<r$.

If $\left\{v_{1}, v_{2}\right\} \cap S^{\prime}=\emptyset$, then clearly $S^{\prime}$ is a cutset in $G$ since $v_{1}$ and $v_{2}$ lie in the same component of $G^{\prime}-S^{\prime}$. So $\kappa(G) \leq\left|S^{\prime}\right|<r \leq \kappa(G)$, a contradiction.

If $\left\{v_{1}, v_{2}\right\} \subseteq S^{\prime}$, then $S=S^{\prime}-v_{1}-v_{2}+v$ is a vertex cut in $G$ and hence $\kappa(G)=$ $|S|=\left|S^{\prime}\right|-1<r-1 \leq \kappa(G)-1$, again a contradiction. 
So we may assume that $\left|\left\{v_{1}, v_{2}\right\} \cap S^{\prime}\right|=1$. Without loss of generality, assume $v_{1} \in S^{\prime}$ and $v_{2} \notin S^{\prime}$. Let $C$ be the component of $G^{\prime}-S^{\prime}$ containing vertex $v_{2}$. If $|C| \geq 2$, then $S=S^{\prime}-v_{1}+v$ is a cutset in $G$ and $\kappa(G) \leq|S|=\left|S^{\prime}\right|<r \leq \kappa(G)$ and once more we have a contradiction. So $|C|=1$; that is, $V(C)=\left\{v_{2}\right\}$. But then $N_{G^{\prime}}\left(v_{2}\right) \subseteq S^{\prime}$ and hence $r \leq\left|N_{G^{\prime}}\left(v_{2}\right)\right| \leq\left|S^{\prime}\right|<r$, and yet again we have a contradiction.

So $\left|S^{\prime}\right| \geq r$ and hence $\kappa\left(G^{\prime}\right)=\left|S^{\prime}\right| \geq r$. But $G^{\prime}$ has a vertex of degree $r$, namely either vertex $v_{1}$ or $v_{2}$, and so $\kappa\left(G^{\prime}\right)=r$ as claimed.

We now are prepared to assemble Lemma 5.2 and the results of the previous two sections in order to prove the following theorem.

Theorem 5.3. Suppose $g$ is any non-negative integer. For each orientable surface $S_{g}$ define the quantity $m_{g}$ by $m_{0}=5, m_{1}=6$ and for $g \geq 2$ by

$$
m_{g}=\left\lfloor\frac{5+\sqrt{1+48\left\lfloor\frac{g}{2}\right\rfloor}}{2}\right\rfloor+1 .
$$

Write $m_{g}=12 s+k$, where $0 \leq k \leq 11$. Then for all integers $i$ in the interval [1, $m_{g}$ ] when $k \neq 2,5$ (respectively, in the interval [1, $m_{g}-1$ ] when $k=2$ or 5 ), there exists a graph $G$ with $\gamma(G)=g$ and $\kappa(G)=i$.

Proof: If $g \leq 11$, the result is immediate by Lemma 5.1.

So assume $g \geq 12$ and hence $s \geq 1$.

Cook [C1] has shown that if $g / 2>0$,

$$
\kappa_{\max }\left(S_{\left\lfloor\frac{g}{2}\right\rfloor}\right)=\left\lfloor\frac{5+\sqrt{1+48\left\lfloor\frac{g}{2}\right\rfloor}}{2}\right\rfloor=m_{g}-1,
$$

and this bound is realized by the complete graph $K_{m}=K_{m_{g}}$. Let $m_{g}=m=12 s+k \geq$ 12 and define $l$ by

$$
l= \begin{cases}g-2 \gamma(s, k)+1, & \text { if } k \neq 2,5 \\ g-2 \gamma(s, k)+3, & \text { if } k=2,5 .\end{cases}
$$

1. Suppose $k=0,3,4$ or 7 .

By performing splitting (see Lemma 5.2) on graph $H_{I}(k, l, m)$, we can saturate the interval $[1,6 s+\lceil(k+1) / 2\rceil$, by Theorems 3.2 and 3.3 we can saturate $[l+2,2 l+2]$, by Theorems 4.7 and 4.6 we can saturate $[2 l+3, m-1]$, and by Theorem 4.8 , we can extend the last interval to $[2 l+3, m]$.

2. Suppose $k=1,6,8,9,10$ or 11 .

By performing splitting on graph $H_{I}(k, l, m)$, again we can saturate the interval $[1,6 s+\lceil(k+1) / 2\rceil]$, by Theorem 3.2 we can saturate interval $[l+2,2 l+1]$ and by Theorem 3.3 we can extend this to $[l+2,2 l+3]$. Again by Theorems 4.7 and 4.6 we can saturate $[2 l+3, m-1]$ and by Theorem 4.8 we can extend this to $[2 l+3, m]$. 
3. Finally suppose $k=2$ or 5 .

In this case, performing splitting on graph $H_{I}(k, l, m)$ yields saturation of $[1,6 s+$ $\lfloor(k+1) / 2\rfloor]$, Theorem 3.2 yields saturation of $[l+2,2 l+1]$ and Theorem 3.3 allows us to extend this to $[l+2,2 l+2]$. Then Theorems 4.7 and 4.6 allow saturation of $[2 l+2, m-2]$ and Theorem 4.8 allows us to extend this to $[2 l+2, m-1]$.

It is routine to check that for $k \neq 2,5$, we have $6 s+\lceil(k+1) / 2\rceil \geq l+1$ and when $k=2,5$, we have $6 s+\lfloor(k+1) / 2\rfloor \geq l+1$. But then we have shown that when $k \neq 2,5$, we can saturate $[1, m]$ and when $k=2$ or 5 , we can saturate $[1, m-1]$.

Finally note that by Theorems 3.2, 3.3, 4.2 and 4.9 , the genus of each graph constructed for this saturation is $g$. This completes the proof of the theorem.

Theorem 5.4. For every integer $g \geq 0$, let $m_{g}$ be defined as in Theorem 5.3. Then when $0 \leq g \leq 9$, for every integer $i$ in $\left[1, \kappa_{\max }\left(S_{g}\right)\right]$, and when $g \geq 10$, for every integer $i$ in $\left[1,\left\lfloor(\sqrt{2} / 2) \kappa_{\max }\left(S_{g}\right)\right\rfloor\right]$, when $m_{g} \not \equiv 2,5(\bmod 12)$ and for every integer $i$ in $\left[1,\left\lfloor 0.7 \kappa_{\max }\left(S_{g}\right)\right\rfloor\right]$ when $m_{g} \equiv 2,5(\bmod 12)$, there exists a graph $G$ with connectivity $i$ which genus embeds in surface $S_{g}$.

Proof: The result is immediate by Lemma 5.1 when $g \leq 11$, so suppose $g \geq 12$.

First suppose that $m_{g} \not \equiv 2,5(\bmod 12)$.

Let us consider the ratio

$$
\frac{m_{g}}{\kappa_{\max }}=\frac{\left\lfloor\frac{5+\sqrt{1+48\left\lfloor\frac{g}{2}\right\rfloor}}{2}\right\rfloor+1}{\left\lfloor\frac{5+\sqrt{1+48 g}}{2}\right\rfloor} .
$$

We want to show that $m_{g} / \kappa_{\max } \geq \sqrt{2} / 2$.

Case 1. Assume $g$ is odd. Let $g=2 t+1$. Then

$$
\frac{m_{g}}{\kappa_{\max }}=\frac{\left\lfloor\frac{5+\sqrt{1+48 t}}{2}\right\rfloor+1}{\left\lfloor\frac{5+\sqrt{49+96 t}}{2}\right\rfloor}=\frac{\left\lfloor\frac{7+\sqrt{1+48 t}}{2}\right\rfloor}{\left\lfloor\frac{5+\sqrt{49+96 t}}{2}\right\rfloor} .
$$

Let us write $48 t+1=a^{2}+b$, where $a, b$ are non-negative integers such that $0 \leq b<2 a+1$. But then

$$
\frac{m_{g}}{\kappa_{\max }} \geq \frac{a+6}{5+\sqrt{2 a^{2}+4 a+47}} \geq \frac{\sqrt{2}}{2},
$$

whenever $a \geq 6$.

Moreover when $1 \leq a \leq 5$, it follows that $g \leq 1$; i.e., $S_{g}$ is the torus. But we already know that we can saturate the entire connectivity interval for the torus. So Case 1 is proved. 
Case 2. Suppose $g$ is even. Say $g=2 t$. Then

$$
m_{g}=\frac{\left\lfloor\frac{5+\sqrt{1+48 t}}{2}\right\rfloor+1}{\left\lfloor\frac{5+\sqrt{1+96 t}}{2}\right\rfloor} .
$$

But $1+96 t<49+96 t$ and so we are done by the argument for Case 1 .

Now suppose $m_{g} \equiv 2,5(\bmod 12)$.

Case 3. First suppose $g$ is odd.

Then proceeding as in Case 1, it may be shown that

$$
\frac{m_{g}-1}{\kappa_{\max }}=\frac{\left\lfloor\frac{5+\sqrt{1+48 t}}{2}\right\rfloor}{\left\lfloor\frac{5+\sqrt{49+96 t}}{2}\right\rfloor} \geq 0.7
$$

for $a \geq 66$.

When $a<66$, it follows that $g<2 t+1 \leq 187$. However, the number of values of $g$ that must be checked can be reduced from 187 to just 9 by recalling that we are in the case in which $m_{g} \equiv 2,5(\bmod 12)$. It follows easily then that the ratio $\left(m_{g}-1\right) / \kappa_{\max } \geq 0.7$ in eight of these cases. It remains only to consider the single case when $g=35$.

It will suffice to construct a graph $G$ which has $\kappa=17$ and genus 35 . But this construction may be carried out in a manner similar to that above.

Case 4. Finally, suppose $g$ is even.

Letting $g=2 t$ and $1+48 t=a^{2}+b$ and proceeding as in Case 3, it may be shown that $m_{g} / \kappa_{\max } \geq 0.7$ for all $a \geq 48$; i.e., for all $g \geq 95$. But there are only six values of $g \leq 94$ for which $m_{g} \equiv 2,5(\bmod 12)$ and one easily checks each of these values directly to show that $\left(m_{g}-1\right) / \kappa_{\max } \geq 0.7$. This completes the proof of Case 4 and the corollary follows.

The next result follows immediately from Theorem 5.4.

Corollary 5.5. For any orientable surface $S_{g}$,

(i) $\kappa_{\text {gen }}\left(S_{g}\right)=\kappa_{\max }\left(S_{g}\right)$, when $0 \leq g \leq 11$,

(ii) $\kappa_{g e n}\left(S_{g}\right) \geq\left\lceil(\sqrt{2} / 2) \kappa_{\max }\left(S_{g}\right)\right\rceil$, when $g \geq 12$ and $m_{g} \not \equiv 2,5(\bmod 12)$, and

(iii) $\kappa_{\text {gen }}\left(S_{g}\right) \geq\left\lceil 0.7 \kappa_{\max }\left(S_{g}\right)\right\rceil$, when $g \geq 12$ and $m_{g} \equiv 2,5(\bmod 12)$. 


\section{The Non-orientable Case}

The constructions in the non-orientable case parallel in spirit those of the orientable case. Therefore, here we will primarily stress the differences between the non-orientable and the orientable cases.

(1) We shall conform to the tradition of considering the six congruence classes modulo 6 for the non-orientable cases and to that end let $m=6 s+k$ where $s$ and $k$ are non-negative integers with $0 \leq k \leq 5$. Let $\bar{\gamma}(s, k)=\bar{\gamma}\left(K_{6 s+k}\right)$.

(2) For each pair of $s$ and $k$, the base embedding $\Psi$ is a triangulation of $N_{\bar{\gamma}(s, k)}$ when $k=0,1,3,4$, and $K_{6 s+k}-K_{2}$ into $N_{\bar{\gamma}(s, k)-1}$ when $k=2,5$ [J1, K1, R1, R2, RY1].

(3) Let $\bar{l}_{k}(s)=2[\bar{\gamma}(s, k+1)-\bar{\gamma}(s, k)]+1$, for $k \neq 2,5$, and $\bar{l}_{k}(s)=2[\bar{\gamma}(s, k+1)-$ $\bar{\gamma}(s, k)]+3$, for $k=2,5$. This number is an upper bound of the number of crosscaps we need to construct the desired non-orientable surfaces.

(4) For $k=2,5$, the base embeddings are not necessarily non-orientable [R1]. In each of these cases, we need at least two tubes to construct the desired surfaces and we always twist the last tube to obtain a non-orientable surface.

(5) If a given non-orientable surface has odd Euler characteristic, when constructing embeddings in this surface we need to add a crosscap on a connecting tube to guarantee that the resulting surface is non-orientable. Also we need to put extra edges on this crosscap that are not parallel to other edges to obtain a genus embedding. The crosscap is added on the last tube for the construction analogous to Construction I and is added on the first tube for the construction analogous to Construction II.

Using arguments similar to those in the orientable cases, we obtain the following results. The proofs are omitted here, but may be checked in an Appendix available on the world wide web [PZ2].

Theorem 6.1. Suppose $h$ is any positive integer. For each non-orientable surface $N_{h}$ define the quantity $m_{h}$ by $m_{1}=6$ and for $h \geq 2$ by

$$
m_{h}=\left\lfloor\frac{5+\sqrt{1+24\left\lfloor\frac{h}{2}\right\rfloor}}{2}\right\rfloor+1 .
$$

Write $m_{h}=6 s+k$, where $0 \leq k \leq 5$. Then for all integers $i$ in the interval [1, $\left.m_{h}\right]$ when $k \neq 2,5$ (respectively, in the interval $\left[1, m_{h}-1\right]$ when $k=2$ or 5 ), there exists a graph $G$ with $\bar{\gamma}(G)=h$ and $\kappa(G)=i$.

Theorem 6.2. For every integer $h>0$, let $m_{h}$ be defined as in Theorem 6.1. Then when $1 \leq h \leq 7$, for every integer $i$ in $\left[1, \kappa_{\max }\left(N_{h}\right)\right]$, and when $h \geq 8$, for every integer $i$ in $\left[1,\left\lfloor(\sqrt{2} / 2) \kappa_{\max }\left(N_{h}\right)\right\rfloor\right]$, when $m \not \equiv 2,5(\bmod 6)$ and for every integer $i$ in $\left[1,\left\lfloor 0.7 \kappa_{\max }\left(N_{h}\right)\right\rfloor\right]$ when $m \equiv 2,5(\bmod 6)$, there exists a graph $G$ with connectivity $i$ which genus embeds in surface $N_{h}$. 
Theorem 6.3. For any non-orientable surface $N_{h}$,

(i) $\kappa_{\text {gen }}\left(N_{h}\right)=\kappa_{\max }\left(N_{h}\right)$, when $1 \leq h \leq 7$,

(ii) $\kappa_{\text {gen }}\left(N_{h}\right) \geq\left\lceil(\sqrt{2} / 2) \kappa_{\max }\left(N_{h}\right)\right\rceil$, when $h \geq 8$ and $m_{h} \not \equiv 2,5(\bmod 6)$, and

(iii) $\kappa_{\text {gen }}\left(N_{h}\right) \geq\left\lceil 0.7 \kappa_{\max }\left(N_{h}\right)\right\rceil$, when $h \geq 8$ and $m_{h} \equiv 2,5(\bmod 6)$.

\section{Concluding Remarks}

It has been shown above that for genus embeddings, one can always saturate a subinterval of the connectivity interval of a surface $\Sigma$, namely the subinterval $\left[1,\left\lfloor 0.7 \kappa_{\max }(\Sigma)\right\rfloor\right]$ (or sometimes on the subinterval $\left[1,\left\lfloor(\sqrt{2} / 2) \kappa_{\max }(\Sigma)\right\rfloor\right]$ ). But what about saturation of the remaining subinterval $\left[\left\lfloor 0.7 \kappa_{\max }(\Sigma)\right\rfloor+1, \kappa_{\text {gen }}(\Sigma)\right]$ ?

For some surfaces (such as $S_{36}$ ), saturation is not possible because $\kappa_{\text {gen }}<\kappa_{\max }$ for these surfaces. In [PZ1] these are called Class $\mathcal{B}$ surfaces and it is shown there that such surfaces must occur periodically infinitely often as the value of the genus goes to infinity.

In the Introduction it was claimed that a complete answer to Question 2 would imply the solution to the problem of determining the genus of the octahedron $O(m)$ for some values of $m$ for which this problem is still currently not solved. We conclude with further explanation of this claim. (We will restrict our discussion to the orientable case.)

We recall some terminology and a few results from [PZ1]. Call surfaces for which $\kappa_{\text {gen }}=\kappa_{\max }$, Class $\mathcal{A}$. Thus since it is clear that $\kappa_{g e n} \leq \kappa_{\max }$ for all surfaces, the set of all surfaces partitions into those which are Class $\mathcal{A}$ and those which are Class $\mathcal{B}$. The Class $\mathcal{A}$ and Class $\mathcal{B}$ genera are nicely arranged in the following sense. Every finite interval of genera of the form $\left[\gamma\left(K_{m}\right)+1, \gamma\left(K_{m+1}\right)\right]$ can be written as the disjoint union of two subintervals $\left[\kappa\left(K_{m}\right)+1, g_{\mathcal{A}}-1\right] \cup\left[g_{\mathcal{A}}, \gamma\left(K_{m+1}\right)\right]$ where the first of the two subintervals consists entirely of Class $\mathcal{B}$ genera and the second entirely of Class $\mathcal{A}$ genera. Moreover, it was shown that if $m \geq 30$, then the Class $\mathcal{B}$ subinterval is not empty. The value of $g_{\mathcal{A}}$ is called the breaking point of the interval $\left[\gamma\left(K_{m}\right)+1, \gamma\left(K_{m+1}\right)\right]$.

We now show that if we knew the complete answer to Question 2, then we could compute the genus of the octahedron $O(m)$ for at least some of the values of $m$ for which it is presently unknown.

Write $m=12 s+k$ where $0 \leq k \leq 11$. In [PZ1] it was shown that for $k=1, \ldots, 7$ and $11, g_{\mathcal{A}}$ for the interval $\left[\gamma\left(K_{m}\right)+1, \gamma\left(K_{m+1}\right)\right]$ must equal to $\gamma(O(m+1))$. (We stress here that this does not mean that we know the numerical value of $\gamma(O(m+1))$ !) Set $i=\kappa(O(m+1))$ in Question 2, where $m=12 s+k$ and assume that $k$ has one of the eight values $1, \ldots, 7$ or 11 . (Clearly $i=m-1$.)

Inspect the set of genera $1,2,3, \ldots$, in increasing order. Among the genera in the interval $\left[1, \gamma\left(K_{m}\right)-1\right]$, none has an $(m-1)$-connected graph which genus embeds there, since $\kappa_{\text {gen }} \leq \kappa_{\max } \leq m-2$ for all these genera. The genus $\gamma\left(K_{m}\right)$ does have an $(m-1)$-connected graph which genus embeds (namely, $K_{m}$ !). Then in the next subinterval $\left[\gamma\left(K_{m}\right)+1, g_{\mathcal{A}}-1\right]$, none of the genera admits the genus embedding of any 
$(m-1)$-connected graph. That is because the genera in this subinterval all correspond to Class $\mathcal{B}$ surfaces and hence $\kappa_{\text {gen }}<\kappa_{\max }=m-1$ for these genera.

The next genus encountered is $g_{\mathcal{A}}$ and since it is the genus of a Class $\mathcal{A}$ surface, it does admit the genus embedding of an $(m-1)$-connected graph. (We do not necessarily know the identity of such a graph, but that is irrelevant for our purposes here.) So starting at genus 1 and allowing the genus to increase by one at each step, since we know the answer to Question 2 at each step, we simply record the second genus encountered where the answer to Question 2 is "yes"; that is, the second genus encountered which admits the genus embedding of some graph having connectivity $m-1$ (the first genus encountered for $\kappa=m-1$ is $\left.\gamma\left(K_{m}\right)\right)$. We know that the numerical value of this genus must be $g_{\mathcal{A}}$. Since we have assumed that $m \equiv 1, \ldots, 7(\bmod 12)$ or $m \equiv 11(\bmod 12)$, by Theorem 5.1 of [PZ1] we know that this numerical value for $g_{\mathcal{A}}$ must be the same

as the value of $\gamma(O(m+1))$. In this way, we have determined the numerical value of $\gamma(O(m+1))$.

We conclude with the following conjecture:

Conjecture: For any integer $i \in\left[1, \kappa_{g e n}(\Sigma)\right]$, there is a graph which genus embeds in $\Sigma$ and has connectivity $i$.

\section{References}

[AG1] S. Alpert and J.L. Gross, Branched coverings of graph embeddings, Bull. Amer. Math. Soc., 79, 1973, 942-945.

[C1] R.J. Cook, Heawood's theorem and connectivity, Mathematika, 20, 1973, 201207.

[D1] R.A. Duke, The genus, regional number, and Betti number of a graph, Canad. J. Math., 18, 1966, 817-822.

[E1] J. Edmonds, On the surface duality of linear graphs, J. Res. Nat. Bur. Standards Sect. B, 121-135.

[E2] A combinatorial representation for polyhedral surfaces, Notices Amer. Math. Soc., 7, 1960, 646.

[GT1] J.L. Gross and T.W. Tucker, Topological Graph Theory, John Wiley \& Sons, New York, 1987.

[J1] M. Jungerman, Orientable cascades and related embedding techniques, Ph.D. Thesis, Dept. of Mathematics, University of California at Santa Cruz, Santa Cruz, California, 1975. 
[J2] M. Jungerman, The non-orientable genus of $O(n)$, Abstract No. 7, Graph Theory Newslett., 5, 1975, 86.

[J3] M. Jungerman, The non-orientable genus of the symmetric quadripartite graph, J. Combin. Theory Ser. B, 26, 1979, 154-158.

[JR1] M. Jungerman and G. Ringel, The genus of the $n$-octahedron: regular cases, J. Graph Theory, 2, 1978, 69-75.

[K1] V.P. Korzhik, A non-orientable triangular embedding of $K_{n}-K_{2}, n \equiv 8(\bmod 2)$, Discrete Math., 141, 1995, 195-211.

[N1] S. Negami, Construction of graphs which are not uniquely and not faithfully embeddable in surfaces, Yokohama Math. J., 33, 1985, 67-91.

[PZ1] M.D. Plummer and X. Zha, On the connectivity of graphs embedded in surfaces, J. Combin. Theory Ser. B, 72, 1998, 208-228.

[PZ2] M.D. Plummer and X. Zha, On the connectivity of graphs embedded in surfaces II: Appendix, available from: http://www.math.vanderbilt.edu/ plummemd/

[R1] G. Ringel, Map Color Theorem, Springer-Verlag, New York, 1974.

[R2] G. Ringel, Wie man die geschlossen nichtorientierbaren Flächen in möglichst wenig Dreiecke zerlegen kann, Math. Ann., 130, 1955, 317-326.

[RY1] G. Ringel and J.W.T. Youngs, Lösung des Problems der Nachbargebiete auf orientierbaren Flächen, Archiv der Mathematik (Basel), 20, 1969, 190-201.

[S1] S. Stahl, Generalized embedding schemes, J. Graph Theory, 2, 1978, 41-52.

[T1] C. Thomassen, The graph genus problem is NP-complete, J. Algorithms, 10, $1989,568-576$.

[W1] A.T. White, Graphs, Groups and Surfaces, North-Holland, Amsterdam, 1984.

[X1] N.H. Xuong, How to determine the maximum genus of a graph, J. Combin. Theory Ser. B, 26, 1979, 217-225.

[Y1] J.W.T. Youngs, Minimal embeddings and the genus of a graph, J. Math. Mech., 12, 1963, 303-315. 